\title{
Editorial: Perturbation of RNA Binding Protein Regulation in Cancer
}

\author{
Yongsheng $\mathrm{Li}^{1 *}$ and Juan $\mathrm{Xu}^{2 *}$ \\ ${ }^{1}$ Key Laboratory of Tropical Translational Medicine of Ministry of Education, College of Biomedical Information and \\ Engineering, Hainan Medical University, Haikou, China, ${ }^{2}$ College of Bioinformatics Science and Technology, Harbin Medical \\ University, Harbin, China
}

Keywords: RNA binding protein, cancer, network, method, survival

\section{Editorial on the Research Topic}

\section{Perturbation of RNA Binding Protein Regulation in Cancer}

RNA-bind proteins (RBPs) are typically types of proteins that bind RNA to play critical roles in development or cancer (Pereira et al., 2017; Mohibi et al., 2019). Recent studies have identified thousands of RBPs and also revealed the dysregulation of RBPs in various kinds of cancer types, such as mutation (Chen et al., 2019), copy number variation (Xu et al., 2019), expression perturbation as well as perturbations of RBP-gene regulation (Zhang et al., 2020).

With the development of high throughput sequencing technology, some recent studies have highlighted precise dysregulated RBPs in specific cancers. Lung cancer is the leading cause of deaths worldwide and dysregulation of RBPs has been found in lung squamous cell carcinoma (LUSC). $\mathrm{Li}$ et al. analyzed the gene expression and clinical information from The Cancer Genome Atlas (TCGA) and observed 300 aberrantly expressed RBPs. These RBPs were mainly associated with mRNA metabolic processes, RNA modification and cancer-related signaling pathways. Moreover, they identified nine RBP genes for constructing a prognostic model in LUSC. In another study, Zhang et al. characterized the clinical relevance of RBPs in colorectal cancer. First, 242 differentially expressed RBPs were identified and eight RBPs were found to be related with the prognoses of colorectal cancer patients. Four RBPs (NOL3, PTRH1, UPF3B, and SMAD6) were used to construct the prognostic risk score model. In addition, Zhong et al. also constructed a prognostic model based on RBP expression in kidney renal clear cell carcinoma. Furthermore, potential drugs for cancer were predicted based on the Connectivity Map database. Moreover, RBPs were also play important roles during cancer progression (Wang et al.).

In addition, although some targets of RBPs were identified based on computational or experimental methods, the genome-wide RBP-gene regulatory network in cancer is largely unknown and little is known about the synergetic interaction between RBPs and other regulators. In recent studies, co-expression network analysis was applied to predict the function of RBPs (Wu et al.). In the past decade, these studies about RBPs mainly focused on mutations in RBPs or their target genes. However, it has been increasing appreciated that many driver mutations might perturb molecular interactions or regulatory networks (Mosca et al., 2015; Yi et al., 2017). Recently, a computational method Mutational Effect on RNA Interactome Topology (MERIT) was proposed to analyze the RBP-gene regulatory networks across cancer types (Li et al., 2019a). All these results provide insights into characterizing perturbed RBP-RNA regulatory networks in cancer, as well as the genotype-phenotype relationships underlying human cancers, and RBPs are potential biomarkers for precision medicine. 
The methylation of N6 adenosine (m6A) plays a critical role in diverse biological processes (Li et al., 2019b). Moreover, recent studies have revealed that RBPs also play important roles in RNA methylation. IGF2BP3 was identified as a potential oncogene across multiple cancer types and also play important roles in tissue development (Xu et al., 2021; Zhang et al., 2021). These studies provide another regulatory layer of RBPs in cancer.

In summary, RBPs play important roles in cancer development and progression. All these integrated analysis provided detailed knowledge of the function of the RBPs in cancer, which will facilitate the development of rational therapies for cancer.

\section{REFERENCES}

Chen, H. J., Topp, S. D., Hui, H. S., Zacco, E., Katarya, M., McLoughlin, C., et al. (2019). RRM adjacent TARDBP mutations disrupt RNA binding and enhance TDP-43 proteinopathy. Brain 142, 3753-3770. doi: 10.1093/brain/awz313

Li, Y. S., McGrail, D. J., Xu, J., Li, J. Y., Liu, N. N., Sun, M., et al. (2019a). MERIT: systematic analysis and characterization of mutational effect on RNA interactome topology. Hepatology 70, 532-546. doi: 10.1002/hep.30242

Li, Y. S., Xiao, J., Bai, J., Tian, Y., Qu, Y. W., Chen, X., et al. (2019b). Molecular characterization and clinical relevance of $\mathrm{m}$ A regulators across 33 cancer types. Mol. Cancer 18:137. doi: 10.1186/s12943-019-1066-3

Mohibi, S., Chen, X. B., and Zhang, J. (2019). Cancer the'RBP'eutics-RNAbinding proteins as therapeutic targets for cancer. Pharmacol. Ther. 203:107390. doi: 10.1016/j.pharmthera.2019.07.001

Mosca, R., Tenorio-Laranga, J., Olivella, R., Alcalde, V., Ceol, A., Soler-Lopez, M., et al. (2015). dSysMap: exploring the edgetic role of disease mutations. Nat. Methods 12, 167-168. doi: 10.1038/nmeth.3289

Pereira, B., Billaud, M., and Almeida, R. (2017). RNA-binding proteins in cancer: old players and new actors. Trends Cancer 3, 506-528. doi: 10.1016/j.trecan.2017.05.003

Xu, K., Cai, Y., Zhang, M., Zou, H., Chang, Z., Li, D., et al. (2021). Pan-cancer characterization of expression and clinical relevance of m6A-related tissue-elevated long noncoding RNAs. Mol. Cancer 20:31. doi: 10.1186/s12943-021-01 $324-8$

\section{AUTHOR CONTRIBUTIONS}

All authors listed have made a substantial, direct and intellectual contribution to the work, and approved it for publication.

\section{FUNDING}

This work was supported by Hainan Provincial Natural Science Foundation of China (Grant No. 820MS053), the National Natural Science Foundation of China (Grant No. 31970646, $61873075,32060152,32070673,31871338$ ), Heilongjiang Touyan Innovation Team Program.

Xu, X. D., Yu, Y., Zong, K., Lv, P. W., and Gu, Y. T. (2019). Up-regulation of IGF2BP2 by multiple mechanisms in pancreatic cancer promotes cancer proliferation by activating the PI3K/Akt signaling pathway. J. Exp. Clin. Cancer Res. 38:497. doi: 10.1186/s13046-019-1470-y

Yi, S., Lin, S. D., Li, Y. S., Zhao, W., Mills, G. B., and Sahni, N. (2017). Functional variomics and network perturbation: connecting genotype to phenotype in cancer. Nat. Rev. Genet. 18, 395-410. doi: 10.1038/nrg.2017.8

Zhang, J. W., Li, S. L., Zhang, L., Xu, J., Song, M. X., Shao, T. T., et al. (2020). RBP EIF2S2 promotes tumorigenesis and progression by regulating MYCmediated inhibition via FHIT-related enhancers. Mol. Ther. 28, 1105-1118. doi: 10.1016/j.ymthe.2020.02.004

Zhang, Y., Xu, S. C., Xu, G., Gao, Y. Y., Li, S., Zhang, K., et al. (2021). Dynamic expression of $\mathrm{m}$ A regulators during multiple human tissue development and cancers. Fronti. Cell Dev. Biol. 8:629030. doi: 10.3389/fcell.2020.629030

Conflict of Interest: The authors declare that the research was conducted in the absence of any commercial or financial relationships that could be construed as a potential conflict of interest.

Copyright (C) $2021 \mathrm{Li}$ and Xu. This is an open-access article distributed under the terms of the Creative Commons Attribution License (CC BY). The use, distribution or reproduction in other forums is permitted, provided the original author(s) and the copyright owner(s) are credited and that the original publication in this journal is cited, in accordance with accepted academic practice. No use, distribution or reproduction is permitted which does not comply with these terms. 